Original research article

\title{
Reproductive life characteristics in females affected with Parkinson's disease and in healthy control subjects - a comparative study on Polish population
}

\author{
M. Nitkowska ${ }^{a, *}$, M. Czyżyk ${ }^{b}$, A. Friedman ${ }^{a}$ \\ a Department of Neurology, Medical University of Warsaw, Mazowiecki Szpital Bródnowski, Warsaw, Poland \\ ${ }^{\mathrm{b}}$ Department of Physiotherapy, Mazowiecki Szpital Bródnowski, Warsaw, Poland
}

\section{A R T I C L E I N F O}

Article history:

Received 22 January 2014

Received in revised form

7 August 2014

Accepted 27 August 2014

Available online 16 September 2014

Keywords:

Parkinson's disease

Reproductive lifespan

Menarche

Menopause

\begin{abstract}
A B S T R A C T
Background: Sex and blood level of sex hormones play a key role not only in the susceptibility to develop Parkinson's disease (PD) but also influence the natural course of the disease. The aim of this study was to compare reproductive lifespan events in females affected with PD and in "non-parkinsonian" age matched subjects and to evaluate whether the whole life endogenous oestrogen level is associated with variables describing the course of the disease. Materials and methods: Reproductive lifespan, age at menarche, age at menopause, gynaecological interventions and parity were compared in 76 women with idiopathic PD and in the age-adjusted control group of 74 subjects. Affected women underwent neurological and psychological assessment. Data were analysed using Mann-Whitney U Test and Spearman Rank Correlation Test.

Results: Women affected with PD had a shorter reproductive lifespan and experienced final menstruation earlier than the control group. Early menopause was reported by $24 \%$ of the patients and only by $16 \%$ of the control subjects. Parkinsonian women reported more commonly the history of surgical menopause. Duration of reproductive lifespan, age at menopause and the type of menopause influenced both motor and cognitive functioning of patients.

Conclusions: There may be a relationship between the lifetime average endogenous oestrogen level and the susceptibility to develop PD. Longer reproductive lifespan resulting in higher "whole life" female sex steroids concentrations may exert a protective effect on central nervous system, resulting in milder course of the disease.
\end{abstract}

(C) 2014 Polish Neurological Society. Published by Elsevier Urban \& Partner Sp. z o.o. All rights reserved.

\footnotetext{
* Corresponding author. Current address: Department of Neurology, Czerniakowski Hospital, Stępińska 19/25, 00-739 Warsaw, Poland. Tel.: +48 223186362; fax: +48 223186362.

E-mail address: martakawulak@gmail.com (M. Nitkowska). http://dx.doi.org/10.1016/j.pjnns.2014.08.004 0028-3843/@ 2014 Polish Neurological Society. Published by Elsevier Urban \& Partner Sp. z o.o. All rights reserved.
} 


\section{Introduction}

Parkinson's disease (PD) is a progressive neurodegenerative disease that affects $0.15 \%$ of the general population. Cases of PD increase steeply with advancing age and are consistently higher in men [1]. These two facts have brought the possible protective meaning of female sex steroids to medical attention. Oestrogens play an extremely important role in human brain development [2]. Female sex hormones may exert their positive effect acting via nuclear and membranous receptors, other neurotransmitters receptors and ion channels [3]. Oestrogens may stimulate neurotrophins synthesis and increase concentrations of antiapoptotic factors [4]. Female sex hormones may also act as antioxidants, which have been proven on human hipoccampal, cultured cells (HT22) [5] and attenuate negative effects of MPTP [6]. It was also proven that oestradiol and oestriol may avert alpha synuclein accumulation as well as destabilize the already formed aggregates [7]. Apart from protective role oestrogens may directly stimulate dopamine synthesis in the striatal cells [8].

Studies on animal models although not unanimous also emphasize the importance of sex hormones for dopaminergic system. The drop of endogenous hormones levels in female monkeys after oophorectomy leads to drop in the number of dopaminergic neurons by about 30\% [9]. Sterilized rodents treated with 17-beta oestradiol showed higher levels of dopamine in striatum in comparison to untreated animals $[10,11]$.

The influence of female sex steroids in humans was broadly discussed with regard to cognitive functioning. Higher oestradiol levels were positively correlated with better scores in tests assessing memory and verbal fluency $[12,13]$.

The influence of endogenous oestrogens in women affected with Parkinson's disease is evaluated in vast majority of cases based on analysis of reproductive life events. The studies of Popat et al. [14], Benedetti et al. [15], Yadav et al. [16], Nicoletti et al. [17], and Cereda et al. [18] on age at last menstrual period, type of menopause, cumulative length of pregnancies, use of exogenous oestrogens and its influence on the risk of developing PD brought contradictory results. In some studies it has been noticed that the age at onset of PD was higher with later age at menopause and longer reproductive lifespan [17-20], while the study of Yadav et al. [16] draws attention to cumulative length of pregnancies as a factor delaying the onset of Parkinson's disease. Another large retrospective study involving women, who underwent uni- or bilateral oophorectomy showed that the risk of all types of parkinsonism was two times higher in comparison to control subjects with no history of gynaecological interventions [21]. On the other hand the study of Simon et al. denied any influence of surgical menopause on the incidence of the disease irrespectively of the age of intervention [22].

The purpose of this study was to compare reproductive life events in women affected with Parkinson's disease and in the age adjusted control group. We also wanted to analyse possible correlations between reproductive lifespan events and selected determinants of the natural course of the disease.

\section{Materials and methods}

Seventy-six females diagnosed with Parkinson's disease treated in the Department of Neurology, of the Medical University of Warsaw and in the outpatients setting were examined. The inclusion criterion in the parkinsonian group was diagnosis of idiopathic Parkinson's disease according to Litvan et al. diagnostic criteria [23]. The exclusion criterion for parkinsonian group was the presence of dementia based on MMSE results as well as the history of surgical interventions (pallidotomy, deep brain stimulation) as the latter could possibly modify the natural course of the disease. The mean age in the PD group was $63 \pm 11$ years (range 42-82), mean duration of the disease was $8 \pm 5$ years (range 1-24), and with an average age of onset being $55 \pm 12$ years. Seventy-four control subjects were randomly selected from the other clinical wards of our hospital. Mean age in the control group was $66 \pm 11$ years (range 50-89). Only women whose records did not contain any documentation of any form of parkinsonism were included to our study. Cognitively impaired females unable to participate in the interview were excluded from the study. Detailed medical history and the exact record of medications use were not obtained from the patients. Those were meaningless for the analysis of past reproductive life events.

Protocol of the study was accepted by the Bioethical Committee of the Medical University of Warsaw. Informed consent was obtained from all patients.

In both groups of patients we conducted a structured interview that contained:

- Age at menarche.

- Age at final menstrual period.

- Type of menopause (natural vs. surgical), as surgical menopause hysterectomy and oophorectomy were taken together.

- Parity meaning cumulative months of pregnancies.

By subtracting the age at menopause and the age at menarche we calculated the duration of reproductive lifespan.

All patients affected with Parkinson's disease underwent neurological and neuropsychological examination by means of different scales:

- Unified Parkinson's Disease Rating Scale (UPDRS) to determine motor and global functioning of the patient in ON and in OFF stage (after $12 \mathrm{~h}$ withdrawal of dopaminergic medication) [24].

- Abnormal Involuntary Movement Scale (AIMS) [25].

- Mini Mental State Examination [26].

- Beck Depression Inventory [27], Parkinson's disease Questionnaire (PDQ39) [28].

We also analysed if any of the reproductive events may influence the determinants of the clinical course of the disease.

Data were analysed with the Mann-Whitney U Test for independent group comparisons and the Spearman Rank Correlation Test for the correlation within the assessed results. 
Table 1 - Reproductive lifespan characteristics in women affected with Parkinson's disease and in the control group.

\begin{tabular}{|c|c|c|c|c|c|}
\hline \multirow[t]{2}{*}{ Females } & \multicolumn{2}{|c|}{$\mathrm{PD}(n=76)$} & \multicolumn{2}{|c|}{ Control group $(n=74)$} & \multirow[t]{2}{*}{$p$} \\
\hline & Mean \pm SD & Range & Mean \pm SD & Range & \\
\hline Age & $63 \pm 11$ & $32-82$ & $67 \pm 11$ & $50-89$ & ns \\
\hline FM & $15 \pm 1$ & 10-19 & $14 \pm 2$ & $11-18$ & ns \\
\hline LM & $47 \pm 5$ & $32-58$ & $50 \pm 5$ & $32-58$ & 0.029 \\
\hline RL & $33 \pm 6$ & $16-43$ & $36 \pm 5$ & $18-46$ & 0.0053 \\
\hline Pregnancies (months) & $12 \pm 8$ & $0-27$ & $19 \pm 10$ & $0-45$ & $<0.001$ \\
\hline
\end{tabular}

All statistical procedures were performed with Statistica (Ver. 9.0 PL). Correlation analysis data were interpreted as: $1-0.7-$ very strong correlation; 0.7-0.5 - strong correlation; $0.5-0.3$ medium strength correlation; 0.3-0.1 - weak correlation positive or negative respectively [29].

\section{Results}

Reproductive life events differed significantly in two groups. Although the mean age at menarche was similar (15 \pm 1 in PD vs. $14 \pm 2$ in controls; $p=n s$ ), the age at last menstrual period was markedly higher in control subjects (mean 50th year of age vs. 47 th year of age in PD patients; $p=0.029$ ) (Table 1 ) $24 \%$ of the parkinsonian patients reported menopause prior to 46th year of age. Although menopause prior to 46th year of age was present only in $16 \%$ of the control subjects, this difference did not reach the statistical significance $(p=0.2)$.

The history of surgical menopause was stated by $39 \%$ of the women affected with Parkinson's disease and only by $16 \%$ of the women included to the control group ( $p=0.002)$.

Reproductive lifespan was markedly shorter in the parkinsonian group than in the control group, on average $33 \pm 6$ years vs. $36 \pm 5$ years in control subjects $(p=0.0053)$.

Cumulative months of pregnancies were higher in the control group than in PD patients $19 \pm 10$ months vs. $12 \pm 8$ months in parkinsonian group $(p=0.000015)$.

Amongst women affected with Parkinson's disease only 7 were in the premenopausal period at the time of examination. None of them reported exacerbation of disease symptoms during menstruation; they also denied fluctuations associated with menstrual cycle.

Majority of patients (77\%) were diagnosed with Parkinson's disease after menopause.

Later age at menopause and longer reproductive lifespan were positively correlated with lower score in the motor part of UPDRS OFF and lower stage in the Hoehn and Yahr OFF stage. It was also positively correlated with lower scores in Beck Depression Inventory (Table 2).

Table 2 - Correlation of age at menopause (LM) and duration of the reproductive lifespan (RL) with UPDRS, H\&Y and BDI scores.

\begin{tabular}{lcc} 
Correlations of two parameters & R-Spearman & $p$ \\
\hline LM and part III UPDRS & -0.30 & 0.01 \\
RL and part III UPDRS & -0.31 & 0.01 \\
RL and BDI & -0.26 & 0.03 \\
\hline
\end{tabular}

Type of menopause - natural vs. surgical - was also important for the natural course of the disease. Women who underwent gynaecological intervention reached higher scores in the UPDRS scale state OFF in comparison to those who did not - on average 40.8 points vs. 31.1 points $(p=0.03)$. They also presented higher score in the motor part of the UPDRS scale in both ON and OFF state -12 vs. $9(p=0.019)$ and 20 vs. 10 $(p=0.005)$ respectively.

Women who had their last menstrual period due to natural reasons were thus in the lower stage of Hoehn and Yahr scale and were able to perform more activities of daily living (higher Schwab and England scale scores) (Table 3).

Weakly expressed difference was also noticeable between women who were diagnosed with Parkinson's disease before menopause and those in whom the disease occurred after the last menstrual period. Although in the first group mentioned mean duration of disease was markedly longer than in the second group (10.7 vs. 7.7; $p=0.02$ ), their global functioning with Parkinson's disease (based on UPDRS ON and OFF scores) was similar to women with later disease onset.

Levodopa dose (in milligrams) needed to achieve similar therapeutic effect was markedly higher in the group affected before the last menstrual period (1152 vs. 797; $p=0.03$ ). Higher levodopa intake lead to severe dyskinesias and higher AIMS score (7.4 vs. 2.0; $p=0.03$ ) in this group of patients. Women affected earlier with PD reached higher PDQ39 scores in comparison to the group with later onset of the disease, which reflects worse general being (Table 4).

\section{Discussion}

The comparison of reproductive lifespan events in healthy women and women affected with Parkinson's disease reveals many differences.

Age at menopause was higher in the control group than in the patients' group resulting in longer reproductive lifespan.

Shorter reproductive lifespan and early age at menopause in women affected with Parkinson's disease were associated with the fact that high percentage of patients underwent gynaecological intervention leading to cessation of menses.

In women affected with Parkinson's disease longer duration of reproductive lifespan was positively correlated with motor functioning. Previous studies of Cereda et al. [18], Ragonese et al. [30] and Haaxma et al. [31] confirm this finding but also point to a relation between longer reproductive lifespan and later age at onset of Parkinson's disease. Our study did not reveal such correlation. Surprisingly the study by Popat et al. [14], as well as large FRAGRAMP multicenter case 
Table 3 - Comparison of the clinical course of the disease depending on the type of menopause (natural vs. surgical).

\begin{tabular}{lccccc} 
& \multicolumn{2}{c}{ GI $(n=29)$} & & \multicolumn{2}{c}{$p$} \\
\cline { 2 - 3 } & Mean \pm SD & Range & & Mean \pm SD & Range \\
\hline UPDRS_OFF & $40.3 \pm 20.8$ & $3.0-92$ & $31.0 \pm 15.8$ & $7.0-71$ \\
UPDRS_ON_part III & $12.4 \pm 6.7$ & $1.0-27.0$ & $8.9 \pm 5.9$ & $0.0-31$ \\
UPDRS_OFF_part III & $19.8 \pm 8.8$ & $2.0-39$ & $14.5 \pm 7.1$ & $3.0-35$ & 0.03 \\
H\&Y_ON & $2.0 \pm 0.6$ & $1.0-3.0$ & $1.6 \pm 0.6$ & $1.0-2.5$ & 0.019 \\
Hi_Y_OFF & $2.4 \pm 0.9$ & $1.0-4.0$ & $1.9 \pm 0.8$ & 0.005 \\
SE_ON & $86.9 \pm 7.1$ & $70.0-100$ & $91.7 \pm 7.4$ & 0.023 \\
SE_OFF & $75.9 \pm 15.5$ & $40.0-100$ & $85.4 \pm 12.8$ & $60-100$ & 0.029 \\
\hline
\end{tabular}

GI, women who underwent surgical menopause; non-GI, women with natural menopause.

Table 4 - Clinical parameters in women diagnosed before and after menopause.

\begin{tabular}{|c|c|c|c|c|c|}
\hline \multirow[t]{2}{*}{ Group } & \multicolumn{2}{|c|}{ Before menopause $(n=19)$} & \multicolumn{2}{|c|}{ After menopause $(n=49)$} & \multirow[t]{2}{*}{$p$} \\
\hline & Mean \pm SD & Range & Mean \pm SD & Range & \\
\hline Duration & $10.7 \pm 5.4$ & $1.0-21$ & $7.7 \pm 5.2$ & $1.0-24.0$ & 0.02 \\
\hline L_DOPA & $1152.6 \pm 577.5$ & $300.0-2250$ & $797.0 \pm 472.6$ & $0.0-2100$ & 0.03 \\
\hline AIMS & $7.4 \pm 8.0$ & $0.0-27.0$ & $2.9 \pm 5.1$ & $0.0-22.0$ & 0.03 \\
\hline PDQ 39 & $56.3 \pm 23.8$ & $9.0-94.0$ & $41.8 \pm 19.2$ & $8.0-76.0$ & 0.02 \\
\hline AIMS/years & $0.6 \pm 0.7$ & $0.0-2.5$ & $0.3 \pm 0.6$ & $0.0-3.0$ & 0.04 \\
\hline UPDRS_ON & $22.3 \pm 10.3$ & $7.0-48$ & $24.8 \pm 14.0$ & $2.0-65.0$ & ns \\
\hline UPDRS_OFF & $39.4 \pm 17.4$ & 12.0 & $34.2 \pm 19.1$ & $3.0-92.0$ & ns \\
\hline
\end{tabular}

control study [17] denied any of the above-mentioned outcomes. The study by Popat et al. [14] has proven that early menopause as well as surgical menopause decreases the risk of developing Parkinson's disease, while the latter study points to the possible influence of exogenous oestrogens intake as a factor increasing the risk of developing Parkinson's disease [17].

Although in the present study we do not deal with disease risk, several results prove positive influence of female endogenous sex hormones. The percentage of women in the parkinsonian group reporting the occurrence of early menopause - prior to 46th year of age - was prominently higher than in control subjects (16\%) (for Polish population average at final menstrual period ranges between 47 th and 52nd year of age) [32]. The cessation of menses due to surgical menopause was also more frequent among affected women than in control subjects. The study of Benedetti et al. [15] brought similar results. Women affected with Parkinson's disease underwent surgical menopause more often in comparison to control subjects (18 vs. $7 \%$ ) and the affected women reported early final menstrual period - prior to 46th year of age.

One of the outcomes of our study, concerning possible protective effects of female sex hormones, might be worth further investigation. Women diagnosed with Parkinson's disease before menopause compared with those diagnosed after menopause despite longer duration of the disease did not differ when assessed with clinical scales estimating motor functioning and cognitive impairment. On the other hand surgical menopause causing very abrupt decrease in oestrogens levels was associated with much worse outcomes in the UPDRS scale in both on and off stages.

At this point it is worth explaining why by surgical menopause we understood all medical procedures leading to cessation of menses - both hysterectomy and uni- or bilateral oophorectomy. It has been proven that hysterectomy may solely impair the level of endogenous oestrogens due to damaging blood support for the ovary. Ipso facto hysterectomy may lead to ovarian failure in several years [33-36]. Greater risk of developing all types of parkinsonism after uni- or bilateral oophorectomy as well as after hysterectomy was also noted by Rocca et al. [21].

The only similarity in reproductive life events in our study in the two groups was the age at menarche. It is therefore inconsistent with the finding of Martignnoni et al. [37] and Cereda et al. [18] who found that age at menarche was markedly higher in the parkinsonian group, compared to healthy subjects and was positively correlated with the age at PD onset respectively.

In the present study in a group of patients who had Parkinson's disease diagnosed prior to the final menstrual period $(n=7)$, neither detailed medical history nor available clinical records revealed any endocrinological abnormalities. None of the patients reported cycle related fluctuations of the disease symptoms. The latter finding is inconsistent with those of Quinn and Marsden [38] who pointed to marked deterioration of parkinsonian symptoms associated with menstruation, which could have been associated with lower endogenous oestrogen level at this time of menstrual cycle.

Cumulative length of pregnancies was longer in the control subjects than in the parkinsonian group. This finding is consistent with the study of Martignnoni et al. [37] and Yadav et al. [16]. The latter study as well as the study of Haaxma et al. associated longer pregnancy with later onset of Parkinson's disease, thus implying its protective mechanism [14]. Our study does not confirm this thesis. Many previous researches point to long cumulative length of pregnancies as a possible risk factor for developing Parkinson's disease [20,30]. Such hypothesis arises from the fact that during pregnancy oestriol 
becomes the most prominent oestrogen in women's body. Its activity and affinity to oestrogen's receptor are much weaker than those of oestradiol [3]; however, studies on cultured cells have proven its great potential to prevent formation of alpha synuclein and to damage the already formed aggregates [7]. It has also been raised that oestriol may protect from glutamate induced excitotoxicity [39]. It is therefore worth reflection whether time of pregnancy with its high oestriol and low oestradiol level may be protective or harmful for dopaminergic system.

\section{Conclusions}

Our study indicates that there may be an association between the lifetime average oestrogen level and the susceptibility to develop Parkinson's disease. Early menopause and shorter reproductive lifespan can increase the risk of developing the disease. The most harmful for functioning of the central nervous system seems to be the surgical menopause leading to abrupt decrease in endogenous oestrogen level. Protective potential of endogenous oestrogens might be also confirmed by milder disease course in women diagnosed before their final menstrual period.

The limitation of our study is the fact that we had to assume the whole life oestrogen level based on the reproductive life events and not on the exact hormone levels. Perhaps the comparison of postmenopausal sex hormones and gonadotropins levels might be of value. Because of difficulties in interviewing demented patients this group was not included into our study, leaving a deficiency, which should be further investigated and perhaps linked to studies on oestrogen influence on cognitive functioning.

Another limitation of our study is lack of medical records of exogenous oestrogens intake in both groups studied. Although only few women reported oral contraceptives/hormonal replacement therapy use (which perhaps is unique in Polish population), they could not remember the medications names, which unabled the identification of drugs components (oestrogens/progesterone solely or mixed preparates) - therefore, we could not include these data into our study. Nevertheless collecting such data in the future, as the medical records are nowadays easier to reach could be of value.

\section{Conflict of interest}

None declared.

\section{Acknowledgement and financial support}

None declared.

\section{Ethics}

The work described in this article has been carried out in accordance with The Code of Ethics of the World Medical Association (Declaration of Helsinki) for experiments involving humans; Uniform Requirements for manuscripts submitted to Biomedical journals.

\section{R E F E R E N C E S}

[1] Van den Eeden SK, Tanner CM, Bernstein AL. Incidence of Parkinson's disease: variation by age, gender and race/ ethnicity. Am J Epidemiol 2003;157:1015-22.

[2] Küppers E, Ivanova T, Karolczak M, Lazarov N, Föhr K, Beyer C. Classical and nonclassical estrogen action in the developing midbrain. Horm Behav 2001;40:196-202.

[3] McEwen BS. The molecular and neuroanatomical basis for estrogen effects in the central nervous system. J Clin Endocrinol Metab 1999;84:1790-7.

[4] Sawada H, Shimohama S. Estrogens and Parkinson's disease - novel approach for neuroprotection. Endocrine 2003;21:77-9.

[5] Behl C, Widmann M, Trapp T, Holsboer F. 17-Beta estradiol protects neurons from oxidative stress-induced cell death in vitro. Biochem Biophys Res Commun 1995;216:473-82.

[6] Li XL, Cheng WD, Li J. Protective effect of estrogen on apoptosis in a cell culture model of Parkinson's disease. Clin Invest Med 2008;31:E258-64.

[7] Hirohata M, Ono K, Morinaga A, Ikeda T, Yamada M. Antiaggregation and fibril-destabilizing effects of sex hormones on alpha-synuclein fibrils in vitro. Exp Neurol 2009;217:434-9.

[8] Pasqualini C, Olivier V, Guibert B, Frain O, Leviel V. Acute stimulatory effect of estradiol on striatal dopamine synthesis. J Neurochem 1995;65:1651-7.

[9] Leranth C, Roth RH, Elswoth JD, Naftolin F, Horvath TL, Redmond Jr DE. Estrogen is essential for maintaining nigrostriatal dopamine neurons in primates: implications for Parkinson's disease and memory. J Neurosci 2000;20:8604-9.

[10] Pasqualini C, Olivier V, Guibert B. Acute stimulatory effect of estradiol on striatal dopamine synthesis. Neurochemistry 1995;65:1651-7.

[11] Tripanichkul W, Jaroensuppaperch EO, Finkelstein DI. Estrogen enhances the number of nigral dopaminergic neurons of adult male mice without affecting nigral neuroglial number and morphology. Neurosci Lett 2008;435:210-4.

[12] Hogervorst E, De Jager C, Budge M, Smith AD. Serum levels of estradiol and testosterone and performance in different cognitive domains in healthy elderly men and women. Psychoneuroendocrinology 2004;29:405-21.

[13] Kampen DL, Sherwin BB. Estradiol is related to visual memory in healthy young men. Behav Neurosci 1996;110 (June):613-7.

[14] Popat RA, Van Den Eeden SK, Tanner CM. Effect of reproductive factors and postmenopausal hormone use on the risk of Parkinson's disease. Neurology 2005;65:383-90.

[15] Benedetti MD, Maragamore DM, Bower JH. Hysterectomy, menopause and estrogen use preceding Parkinson's disease: an exploratory case control study. Mov Disord 2001;16:830-7.

[16] Yadav R, Shukla G, Goyal V, Behari M. A case control study of women with Parkinson's disease and their fertility characteristics. J Neurol Sci 2012;319(1-2):135-8.

[17] Nicoletti A, Nicoletti G, Arabia G, Annesi G, De Mari M, Lamberti P, et al. Reproductive factors and Parkinson's disease: a multicenter case-control study. Mov Disord 2011;26(14):2563-6.

[18] Cereda E, Barichella M, Cassani E, Caccialanza R, Pezzoli G. Reproductive factors and clinical features of Parkinson's disease. Parkinsonism Relat Disord 2013;19(12):1094-9. 
[19] Galvin JE, Pollack J, Morris JC. Clinical phenotype of Parkinson's disease dementia. Neurology 2006;67:1605-11.

[20] Ragonese P, D'Amelio M, Callari G. Age at menopause predicts age at onset of Parkinson's disease. Mov Disord 2006;21:2211-4.

[21] Rocca WA, Bower JH, Maraganore DM. Increased risk of parkinsonism in women who underwent oophorectomy before menopause. Neurology 2008;70:200-9.

[22] Simon KC, Chen H, Gao X, Schwarzschild MA, Ascherio A. Reproductive factors, exogenous estrogen use, and risk of Parkinson's disease. Mov Disord 2009;24: 1359-65.

[23] Litvan I, Bhatia KP, Burn DJ, Goetz CG, Lang AE, McKeith I, et al. Movement Disorders Society Scientific Issues Committee report: SIC Task Force appraisal of clinical diagnostic criteria for Parkinsonian disorders. Mov Disord 2003;18:467-86.

[24] Movement Disorder Society Task Force on Rating Scales for Parkinson's disease. UPDRS: status and recommendations. Mov Disord 2003;18:738-50.

[25] Goetz CG. Rating scales for dyskinesias in Parkinson's disease. Mov Disord 1999;14(Suppl. 1):48-53.

[26] Folstein MF, Folstein SE, McHugh PR. Mini-mental state: a practical method for grading the state of patients for the clinician. J Psychiatr Res 1975;12:189-98.

[27] Beck AT, Ward CH, Mendelson M. An inventory for measuring depression. Arch Gen Psychiatry 1961;4: $561-7$.

[28] Jenkinson C, Peto V, Fitzpatrick R, Greenhall R, Hyman N. Self reported functioning and well being in patients with Parkinson's disease: comparison of the Short Form Health Survey (SF-36) and the Parkinson's Disease Questionnaire (PDQ-39). Age Ageing 1995;24:505-9.
[29] Cohen J. Statistical power analysis for the behavioral sciences. 2nd ed. Lawrence Erlbaum Associates; 1988, ISBN13: $978-0805802832$.

[30] Ragonese P, D'Amelio M, Salemi G. Risk of Parkinson disease in women - effect of reproductive characteristics. Neurology 2004;62:2010-4.

[31] Haaxma CA, Bloem BR, Borm GF. Gender differences in Parkinson's disease. J Neurol Neurosurg Psychiatry 2007;78:819-24.

[32] Kaczmarek M. Determination of the age of menopause in Polish women. Przegl Menop 2007;2:77-82 [in Polish].

[33] Siddle N, Sarrel P, Whitehead M. The effect of hysterectomy on the age of ovarian failure: identification of a subgroup of women with premature loss of ovarian function in literature review. Fertil Steril 1986;47:94-100.

[34] Bukovsky I, Halperin R, Schneider D. Ovarian function following abdominal hysterectomy with and without unilateral oophorectomy. Eur J Obstet Gynecol Reprod Biol 1995;58:29-32

[35] Lindsay MK, Usher DJ. Unrecognized ovarian failure after hysterectomy. Br J Gen Pract 1992;42:529-30.

[36] Stone SC, Dickey RP, Mickal A. The acute effect of hysterectomy on ovarian function. Am J Obstet Gynecol 1977;127:349-52.

[37] Martignnoni E, Nappi RE, Citterio A. Reproductive life milestones in women with Parkinson's disease. Funct Neurol 2003;18:211-7.

[38] Quinn NP, Marsden CD. Menstrual related fluctuations in Parkinson's disease. Mov Disord 1986;1:85-7.

[39] Kajta M, Budziszewska B, Marszal M, Lason W. Effects of 17beta estradiol and estriol on NMDA-induced toxicity and apoptosis in primary cultures of rat cortical neurons. J Physiol Pharmacol 2001;52:437-546. 